\title{
A TIE POINT MATCHING STRATEGY FOR VERY HIGH RESOLUTION SAR-OPTICAL STEREOGRAMMETY OVER URBAN AREAS
}

\author{
C. P. Qiu ${ }^{\mathrm{a}}$, M. Schmitt ${ }^{\mathrm{a}}$, X. X. Zhu ${ }^{\mathrm{a}, \mathrm{b}}$ \\ ${ }^{a}$ Signal Processing in Earth Observation, Technical University of Munich (TUM), Arcisstr. 21, 80333 Munich, Germany - \\ (chunping.qiu, m.schmitt)@tum.de \\ ${ }^{\mathrm{b}}$ Remote Sensing Technology Institute (IMF), German Aerospace Center (DLR), Oberpfaffenhofen, 82234 Wessling, Germany - \\ xiao.zhu@dlr.de
}

KEY WORDS: synthetic aperture radar (SAR), optical imagery, remote sensing, data fusion, stereogrammetry, photogrammetry, radargrammetry, 3D reconstruction, multi-sensor image matching

\begin{abstract}
:
This paper presents a matching strategy for sparsely distributed tie points, which can be used to carry out SAR-optical stereogrammetry. Since matching homologue points in heterogeneous multi-sensor image of complex terrain remains a challenging task, the proposed procedure combines the tie point matching step with the estimation of the corresponding 3D object coordinates. The results of this study are two-fold: On the one hand, they show that fully automatic SAR-optical stereogrammetry using very high resolution data of urban scenes is generally feasible and can provide 3D accuracies in the meter-domain. On the other hand, they highlight the difficulty of multi-sensor image matching, as well as the importance of robust matching strategies.
\end{abstract}

\section{INTRODUCTION}

3D reconstruction of urban areas is of great importance for many different vital tasks, e.g. city planning, autonomous navigation and emergency response. Luckily, today we are living in a world rich of different remote sensing data, with increasingly high resolution and positioning accuracy. Examples include the Worldview missions equipped with the latest optical technology and the TerraSAR-X/TanDEM-X mission, which provides state-ofthe-art synthetic aperture radar (SAR) data with meter-resolution. Besides, both optical and SAR remote sensing provide us with high-quality observations that can be used to map the world's topography (Capaldo et al., 2012, Krieger et al., 2007). Unfortunately, however, every kind of data and the corresponding method for 3D reconstruction has its own inherent limits: highly coherent image pairs from similar viewing angles are a must for any interferometric SAR techniques; cloudy weather causes failure for any optical sensors. So, when used only within mission specifications, the huge earth observation data archive will never be fully exploited. On the other hand, specifically in situations where rapid mapping is required urgently, it would be beneficial to be able to make use of any incoming image, no matter which sensor technology was used to acquire them. As described in (Schmitt and Zhu, 2016b), the solution to this problem is the development of methods for flexible multi-sensor data fusion.

Therefore, this paper investigates SAR-optical stereo reconstruction using sparsely distributed tie points in very high resolution (VHR) spaceborne multi-sensor image of densely built-up urban areas. SAR-optical stereogrammetry has first been proposed by (Bloom et al., 1988), who investigated the general feasibility of the concept using data provided by the SIR-B mission and the Landsat-4/5 satellites. Further investigations were carried out by Raggam et al. (Raggam and Almer, 1990, Raggam et al., 1993, Raggam et al., 1994), who combined Seasat and SPOT/Landsat image for rough DEM generation. Similar experiments using ERS-2/Radarsat-1 and SPOT data were also presented by (Xing et al., 2008). All these studies have shown errors in the dekameter- domain, which seem to prohibit an application of SAR-optical stereogrammetry in the context of urban remote sensing. Only recently, (Zhang et al., 2015) showed that TerraSAR-X and GeoEye1 image can be used to carry out 3D reconstruction with an error in the meter-domain - although the study only used the manually measured corners of a simple-shaped building to proof the concept. In contrast, based on the preliminary considerations sketched in (Schmitt and Zhu, 2016a), our work intends to provide a first step towards a solution of the non-trival problem of automatic stereo matching of VHR multi-sensor images of complex urban areas. In this context, it combines SAR-optical stereogrammetric forward intersection with a fully automatic yet robust selection of sparsely distributed tie points in a simultaneous manner.

\section{SAR-OPTICAL STEREOGRAMMETRY}

In this section, the workflow for SAR-optical stereogrammetry for sparsely distributed tie points located in complex urban areas is described. It is based on the rationale to simultaneously solve the matching and the 3D-reconstruction problems in order to reduce the probability for false matches and thus to enhance the achievable 3D-reconstruction accuracy.

\subsection{A Matching Strategy for Robust Selection of Tie Points}

Since the correct matching of homologue points is a very difficult task for very high resolution multi-sensor remote sensing images, we propose a procedure that incorporates light prior knowledge about the scene of interest and a constraint inspired by epipolar geometry. The algorithm for tie point selection is sketched in Fig. 1. It consists of the following steps:

1. First, a set of keypoints $(r, c)_{s}$ is detected in the SAR image using a block-based Harris corner detector (Ye et al., 2017). Beginning with key points detection in the SAR image instead of the optical image is meant to avoid the situation 


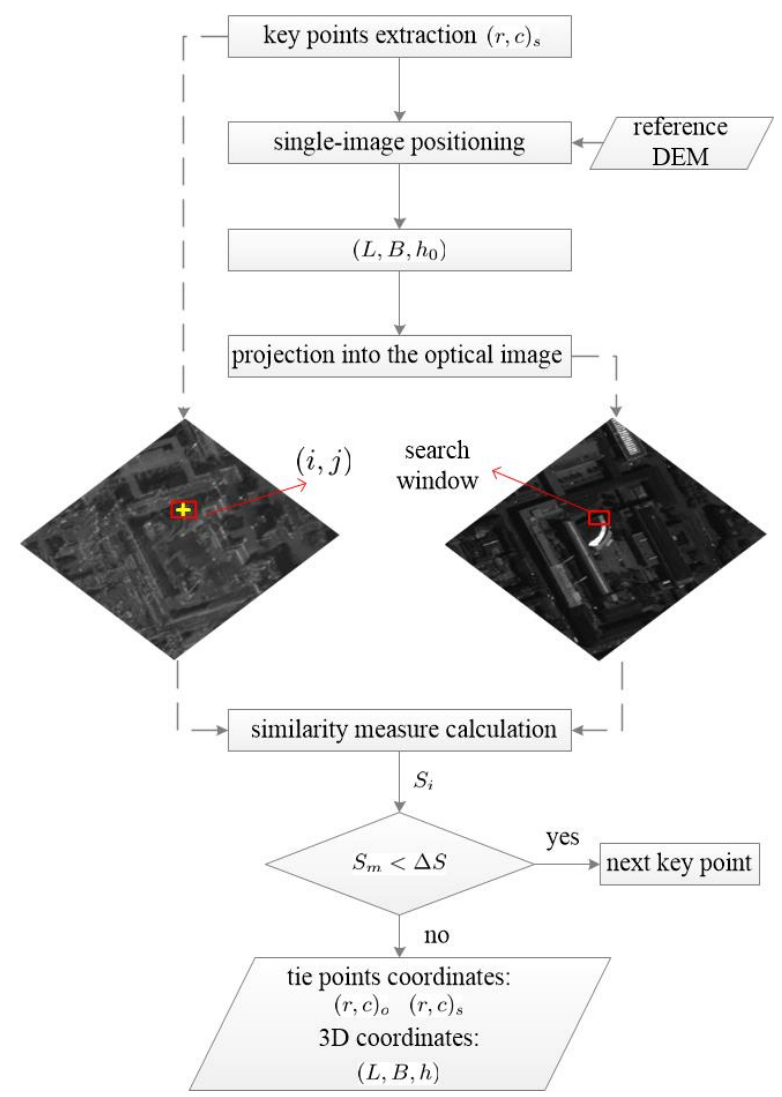

Figure 1. Procedure of the proposed tie point matching strategy.

where a ground point is not existing in the SAR image at all, because of radar shadow or layover.

2. The detected keypoints are then projected onto a coarse terrain model (e.g. the SRTM DEM) using the classical singleimage positioning, yielding approximate ground coordinates $\left(L, B, h_{0}\right)$ for every keypoint. For cities residing on rather flat topography and containing mostly smaller buildings, a simple ground plane located on the mean terrain height can be used as coarse terrain model.

3. For every projected keypoint, $\left(L, B, h_{0}\right)$ is then projected into the optical image, yielding $(r, c)_{o}$. And a search window around this resulting image point is established (the details about this search window are described in Section (2.2).

4. A similarity measure is used to compare all pixels in this search window to the keypoint. Then the similarity measure $S_{i}$ for each pixel $i(i=1,2,3 \ldots N)$ (where $\mathrm{N}$ is the total number of the pixels in the search window) is calculated. When $i=N$, the maximum similarity measure $S_{m}=\max \left(S_{i}\right)$ is determined. This maximum similarity value is then checked against a threshold $\Delta S$ in order to exclude key points for which even the most similar tie point is not a valid match. Thus, if $S_{m}<\Delta S$, the corresponding key point is deleted; otherwise the image point corresponding to the maximum similarity measure, $(r, c)_{o}^{m}$ is considered as the homologue point of the keypoint detected in the SAR image in the first place.

The output of this procedure is the corresponding image coordinates $(r, c)_{o}$ and $(r, c)_{s}$, and the 3D coordinates of this pair of tie points $(L, B, h)$.

\subsection{IMBLS Search Window}

The epipolar-line constraint is well-known in classical photogrammetry (Zhang et al., 1995) and serves as an excellent search strategy, reducing the search space from two dimensions to one dimension. Unfortunately, a rigorous epipolar-line does not exist for SAR stereo pairs (Gutjahr et al., 2014), and even less so for SAR-optical stereo pairs. Therefore, in this paper, we propose a similar search strategy, called Imaging-Model-based-Line-Shape (IMBLS) search window for SAR-optical image matching. As shown in the following section, this strategy can be derived by theoretical considerations of the imaging geometry.

2.2.1 Derivation of the IMBLS Search Window For simplicity and generality, the imaging models of SAR and optical images, respectively, can be expressed using the general non-linear functionals

$$
\left\{\begin{array}{l}
r_{s}=f_{1}\left(\mathbf{p}_{\mathbf{s}} ; L, B, h\right) \\
c_{s}=f_{2}\left(\mathbf{p}_{\mathbf{s}} ; L, B, h\right)
\end{array}\right.
$$

and

$$
\left\{\begin{array}{c}
r_{o}=f_{3}\left(\mathbf{p}_{\mathbf{o}} ; L, B, h\right) \\
c_{o}=f_{4}\left(\mathbf{p}_{\mathbf{o}} ; L, B, h\right),
\end{array}\right.
$$

where $\left(r_{s}, c_{s}\right)$ are tie point coordinates in the SAR image, $\mathbf{p}_{\mathbf{s}}$ are the SAR orientation parameters, $\left(r_{o}, c_{o}\right)$ are the tie point coordinates in the optical image, and $\mathbf{p}_{\mathbf{o}}$ are the optical orientation parameters. $(L, B, h)$ are the $3 \mathrm{D}$ coordinates of the ground point corresponding to this pair of tie points. The derivation starts from the assumption that a keypoint $(r, c)_{s}$ has been detected in the SAR image for which the corresponding point $(r, c)_{o}$ in the optical image needs to be found in order to perform stereogrammetric forward intersection. Taking both steps, i.e. image matching and $3 \mathrm{D}$ reconstruction into consideration simultaneously, the problem can be formulated as a system of four equations with five unknowns: the object coordinates $(L, B, h)$ and the optical tie point image coordinates $(r, c)_{o}$. Although a solution is not possible in a straight-forward manner, a constraint between the unknowns can be constructed as follows. First, (1) and (2) can be rewritten as

$$
\left\{\begin{array}{l}
L=g_{1}\left(\mathbf{p}_{\mathbf{s}} ; h, r_{s}, c_{s}\right) \\
B=g_{2}\left(\mathbf{p}_{\mathbf{s}} ; h, r_{s}, c_{s}\right)
\end{array}\right.
$$

and

$$
\left\{\begin{array}{c}
h=g_{3}\left(\mathbf{p}_{\mathbf{o}} ; L, B, r_{o}\right) \\
h=g_{4}\left(\mathbf{p}_{\mathbf{o}} ; L, B, c_{o}\right) .
\end{array}\right.
$$

where $g_{1}$ and $g_{2}$ describe the mapping of the keypoint coordinates $(r, c)_{s}$ to a spatial position $(L, B)$ for a given height $h$, and $g_{3}$ and $g_{4}$ in analogy are nonlinear functions to derive the height $h$ if the spatial position $(L, B)$ and the optical coordinates $(r, c)_{o}$ are known.

Replacing the ground coordinates $L$ and $B$ in (4) by (3) and combining the two equations inside (4), we get the equality

$$
g_{3}\left(\mathbf{p}_{\mathbf{o}} ; g_{1}, g_{2}, r_{o}\right)=g_{4}\left(\mathbf{p}_{\mathbf{o}} ; g_{1}, g_{2}, c_{o}\right) \text {. }
$$

Finally, from (5) we can acquire the constraint $G$ on the row coordinate $r_{o}$ and the column coordinate $c_{o}$ of the homologue point in the optical image by

$$
r_{o}=G\left(\mathbf{p}_{\mathbf{o}}, \mathbf{p}_{\mathbf{s}} ; c_{o}, r_{s}, c_{s}, L, B, h\right),
$$

where $G$ describes the constraint on the relative position of $r_{o}$ and $c_{o}$. Even though we are not deriving a concise analytic ex- 
pression for the constraint $G$, it shows that a constraint similar to the epipolar constraint can be formulated for any given key point in the SAR image. The constraint could be derived in a similar manner when starting from key points detected in the optical image. In the following, the constraint is used to optimize the search space during the matching process. We use it to construct the IMBLS search window.

2.2.2 Construction of the IMBLS Search Window Exploiting the constraint derived in Section 2.2, a line-shaped search window can be constructed. As Fig.2(a) shows, the procedure starts from an arbitrary key point $(r, c)_{s}$ detected in the SAR image. Using equation (3), and a suitable height search space $h_{k} \in\left[h_{\min }, h_{\max }\right]$, a set of potential spatial ground coordinates $(L, B)_{k}$ can be calculated for every $h_{k}$, and the corresponding optical image coordinates $(r, c)_{k}^{o}$ can be calculated using equation (2). This basic principle is illustrated in Fig.2(b), while the resulting search line described by $(r, c)_{k}^{o}$ can be seen in Fig.2(c). In theory, the tie point corresponding to the original SAR key point needs to be located along this line. However, in order to deal with possible inaccuracies in the imaging parameters $\mathbf{p}_{\mathbf{o}}$ and $\mathbf{p}_{\mathbf{s}}$, a pre-defined buffer width needs to be added around the line. An exemplary IMBLS search window and a regular square search window around the candidate homologue point in the optical image are shown in Fig. 3. Because of the line shape and the fact that the principle is based on the sensor-specific imaging models, it is thus called Imaging-Model-based-Line-Shape search window (IMBLS). Besides a reduced search space, an important advantange of the IMBLS window is that every candidate point located in the window corresponds to a set of 3D coordinates $(L, B, h)$, which means that 3D-reconstruction of object coordinates is solved simultaneous to tie point matching.

\section{EXPERIMENTS AND RESULTS}

To validate the proposed algorithm, experiments with real remote sensing data are carried out.

\subsection{Test Data}

For the experiments presented in this paper, two very high resolution spaceborne datasets over the city of Munich, Germany, have been used. The optical data used is a panchromatic image acquired by Worldview- 2 on July 22, 2015 with mean off-nadir angle $\theta=13.7^{\circ}$ and a mean pixel spacing (ground sampling distance) of $0.49 \mathrm{~m}$. The SAR data used is a TerraSAR-X image acquired in $300 \mathrm{MHz}$ high resolution spotlight mode with an original pixel spacing of $85 \mathrm{~cm}$ in azimuth and $45 \mathrm{~cm}$ in range direction. The SAR image has been filtered by nonlocal NL-SAR filter (Deledalle et al., 2015) and converted to logarithmic scale. Besides the pixel spacing of the SAR image was approximately squared to $0.49 \mathrm{~m}$ in azimuth and slant range for the similarity with the optical image. For the same reason, a rotation was also performed for the SAR image in order to align it coarsely to the orientation of the optical image.

For numerical evaluation of the stereogrammetry result, a dense LiDAR reference point cloud of the same area was used, the parameters of this LiDAR can be found in Tab. 1 (Hebel and Stilla, 2007).

\subsection{Experimental Setup}

For an investigation of the suitability of the matching strategy proposed in this paper, the simplest similarity measure was used:

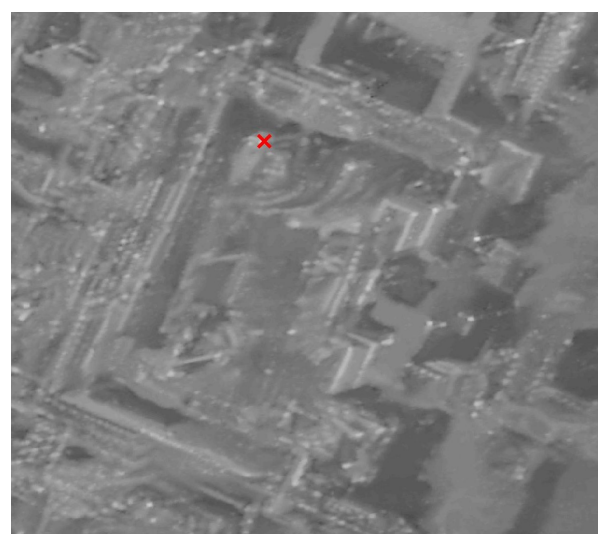

(a) An exemplary key point detected in the SAR image.

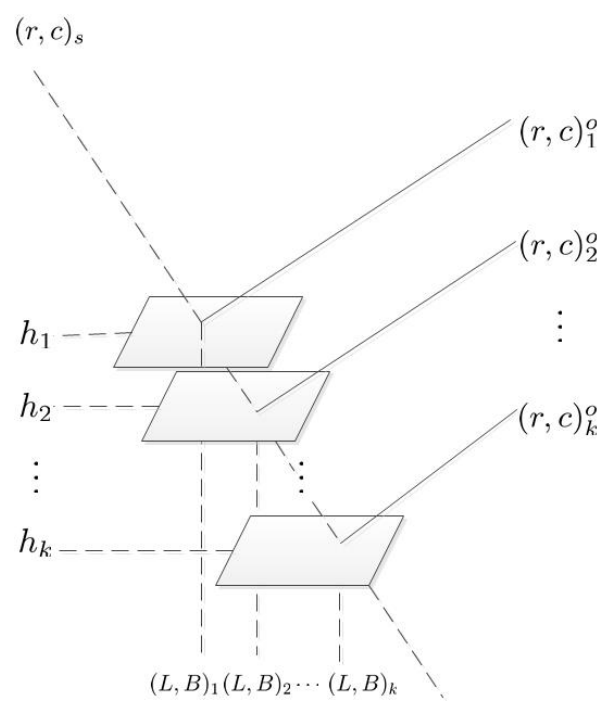

(b) The procedure to calculate the coordinate in the optical image corresponding to every height $h_{k}$.

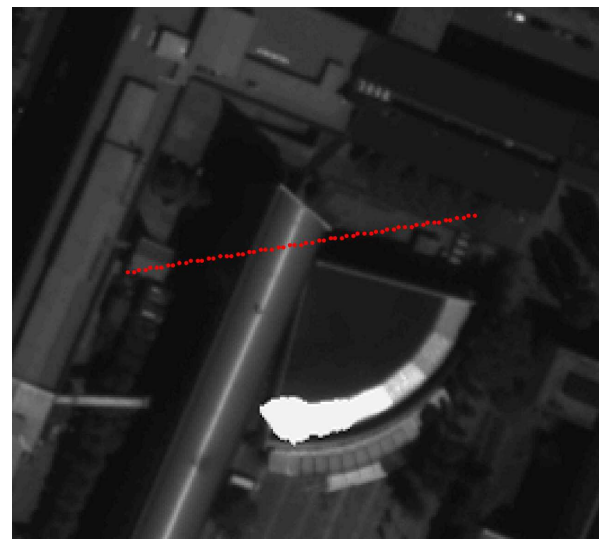

(c) The projection line described by $(r, c)_{k}^{o}$ in the optical image.

Figure 2. Exemplary illustration of the procedure for construction of the IMBLS search window.

normalized cross correlation (NCC). After key point detection, the matching step described in Section 2.2 was carried out. From all keypoints, the $80 \%$-quantile of the highest similarity values were kept to determine the similarity threshold $\Delta S$, i.e. 471 out of 589 originally detected keypoints were considered in the following evaluation. Correspondingly, $\Delta S$ was set to 0.13794 . 


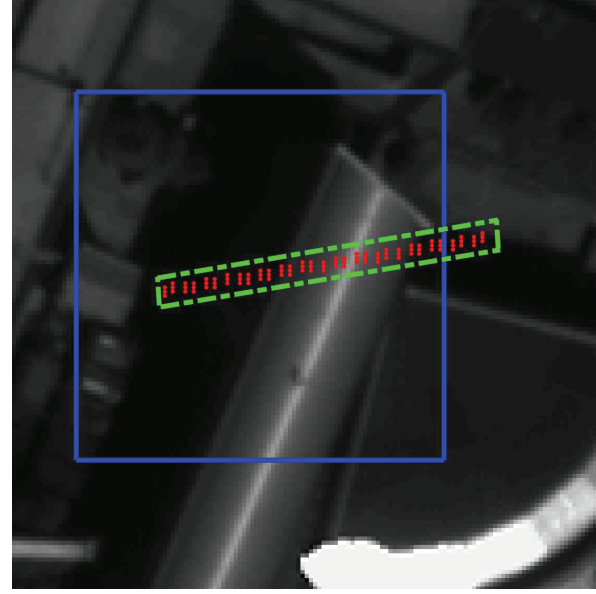

Figure 3. The IMBLS search window (green) and a classical, rectangularly-shaped search window (blue) around the candidate homologue point. (The red dots are the candidates corresponding to every $h_{k}$.)

Table 1. Parameters of the LiDAR reference data.

\begin{tabular}{cc}
\hline \multicolumn{2}{c}{ LiDAR Reference Data } \\
\hline Date of acquisition & 02.09 .2009 \\
Aspects & 4 \\
Number of points & $4,400,000$ \\
Point density & $5.2 \mathrm{pts} / \mathrm{m}^{2}$ \\
\hline
\end{tabular}

The height interval for constructing the IMBLS window was set to $\left[h_{0}-5 m, h_{0}+20 m\right]$, where $h_{0}$ was taken from the SRTM DEM of the study area. $A \pm 1$ pixel pre-defined buffer in the row direction was used to form the final IMBLS search window.

\subsection{Results of Tie Point Matching}

Tie points matching results for the whole study area can be seen in Fig. 4. A zoom-in on the area of the TUM main campus can be seen in Fig. 5 .

\subsection{Results of Stereogrammetric 3D-Reconstruction}

The stereogrammetric 3D-reconstruction accuracy can be evaluated by calculating the distance of each reconstructed 3D point to a LiDAR reference point cloud. To avoid biased results caused by mismatches between the reconstructed $3 \mathrm{D}$ points and the LiDAR data, the distance is not calculated based on individual point neighbors, but based on a plane fitted rigorously through either the 3 nearest neighbors or fitted in a least-squares sense through the 10 nearest neighbors (Schmitt, 2014).

The evaluation results can be found in Tab. 2, which shows both the mean and median of the absolute distances calculated before.

Table 2. The statistic result of the $3 \mathrm{D}$ reconstruction accuracy. (unit: meter)

\begin{tabular}{cccc}
\hline similarity measure & distance & mean & median \\
\hline \multirow{2}{*}{ NCC } & nearest plane & 2.45 & 1.53 \\
& nearest LS plane & 2.97 & 2.06 \\
\hline
\end{tabular}

The visualization of the reconstruction result overlayed to the texturized LiDAR reference point cloud is shown in Fig. 6. To investigate the distribution of these distances in Tab. 2, the histogram of the distances is shown in Fig. 7.

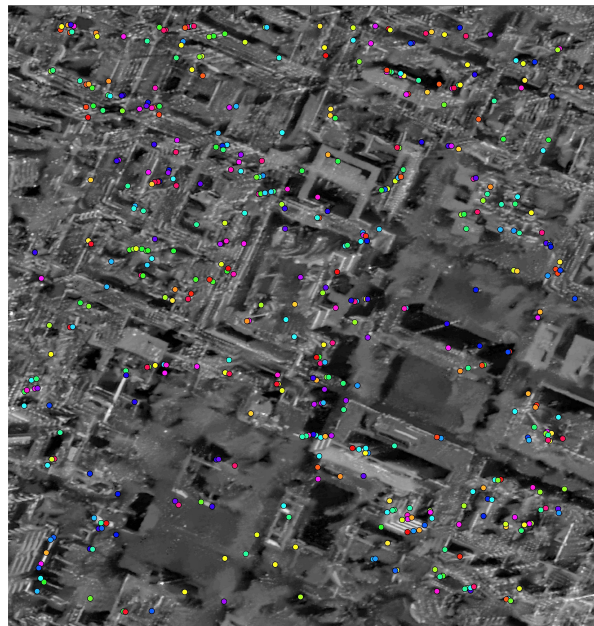

(a) SAR image.

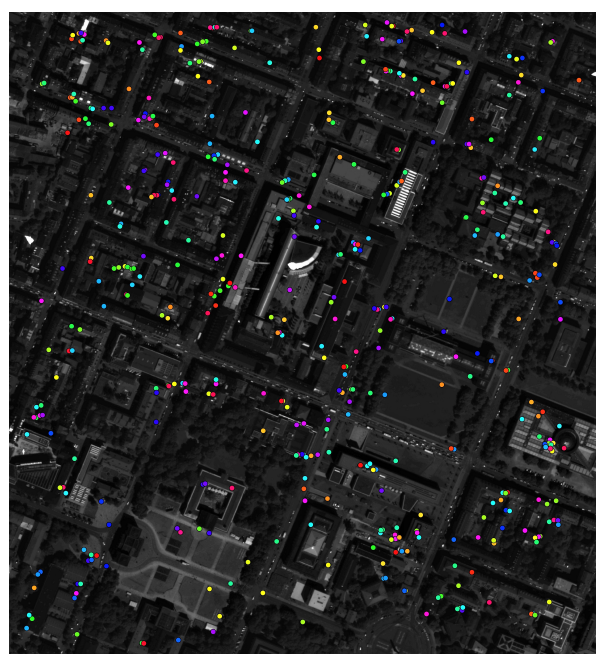

(b) Optical image.

Figure 4. Tie points matching result for the whole study area (Number of tie points: 471).

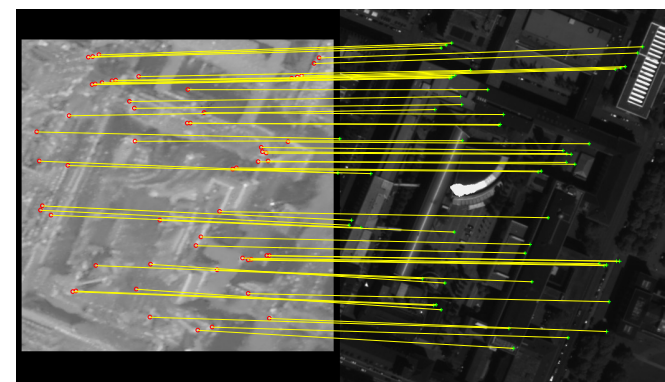

key points in the SAR image Matched points in the optical image

Figure 5. Zoom-in of Fig. 4 to the area of the TUM main campus (Number of tie points: 56 ).

\section{DISCUSSION}

The experimental results presented in Section 3 show that it is generally feasible to carry out stereogrammetric 3D-reconstruction based on very high-resolution SAR and optical imagery, even for complex urban scenes. As the values in Tab. 2 and the histograms in Fig. 7 show, the majority of the keypoints could be reconstructed with reasonable accuracy. Nevertheless, a signifi- 


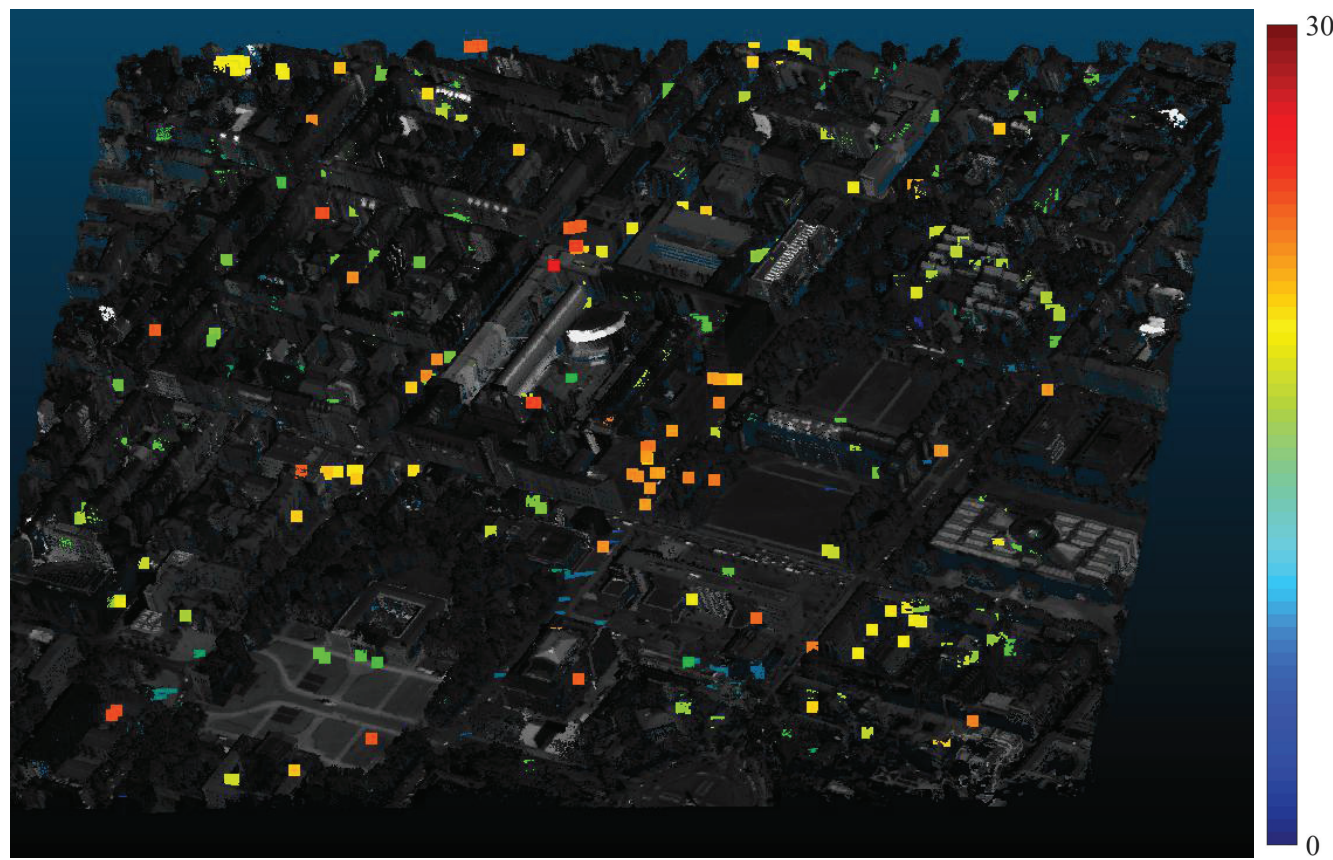

Figure 6. Visualization of the reconstruction result overlayed to the texturized LiDAR reference point cloud. The reconstructed points are colorized by their heights relative to the 560-meter horizontal plane in the UTM system.
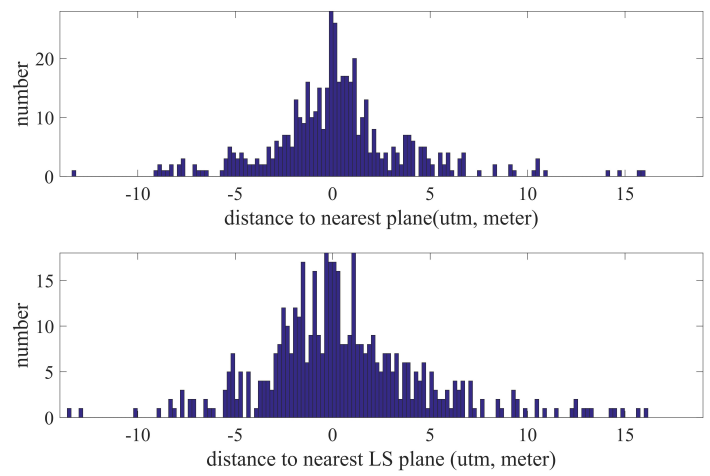

Figure 7. Histogram of the distances corresponding to Tab. 2.

cant amount of points can be considered as erroneous reconstructions as they show 3D distances larger than a couple of meters. Ignoring the potential error budget of the sensor imaging parameters, the only possible reason for erroneous 3D reconstructions is mismatched tie points. This will be detailed in the following section.

\subsection{Effect of Mismatching Errors}

As mentioned before, the major challenge in SAR-optical stereogrammetry is the step of tie point matching in a non-trivial multisensor environment. Figure 8 illustrates, how quickly similaritybased matching can fail if the search window is too large and thus allows ambiguous results.

For discussing the effect of mismatches on the 3D-reconstruction results, we resort to the following procedure, which exploits both the known sensor imaging parameters as well as the LiDAR reference data:

1. In a given pair of correct tie points, which are determined based on the LiDAR reference data, the SAR image coordinates $(r, c)_{s}$ are fixed, and an investigation window of

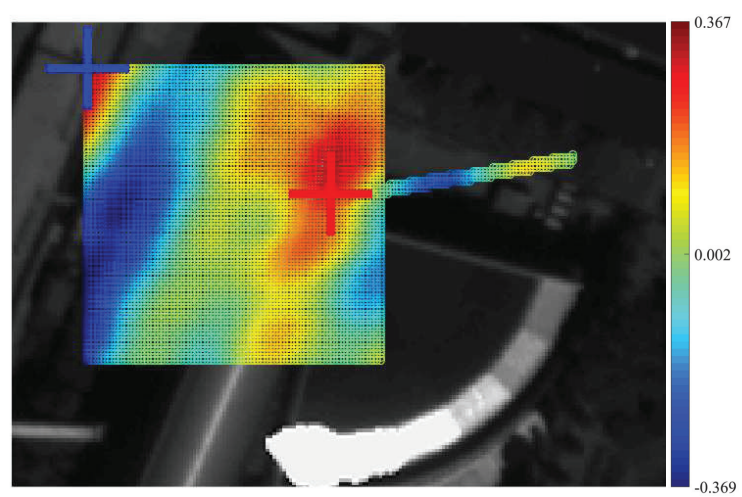

Figure 8. NCC-similarity values within the windows shown in Fig. 3. The red and blue "+"-marks indicate the pixels corresponding to the maximum similarity within the classical rectangular window (blue) and the IMBLS window (red), respectively.

pre-defined size is set up around the homologue point in the optical image $(r, c)_{o}$. All pixels but the center pixel in this investigation window correspond to a mismatch error $(d r, d c)_{(i, j)}=(r, c)_{(i, j)}-(r, c)_{o}$.

2. Together with $(r, c)_{s}$, every pixel $(r, c)_{(i, j)}$ in this window is used for stereogrammetric forward intersection, yielding $(X, Y, h)_{(i, j)}$.

3. Every resulting 3D-coordinate $(X, Y, h)_{(i, j)}$ is then compared to the correct 3D coordinate of this pair of tie points $(X, Y, h)$, yielding the element-wise differences $(d X, d Y, d h)_{(i, j)}=\left|(X, Y, h)-(X, Y, h)_{(i, j)}\right|$, as well as the spatial distance between $(X, Y, h)_{(i, j)}$ and $(X, Y, h), \operatorname{Dis}_{(i, j)}=\left\|(d X, d Y, d h)_{(i, j)}\right\|$.

The result of this simulation can be seen in Fig. 9. It becomes evident that a \pm 10 pixels mismatch will lead to approximately 

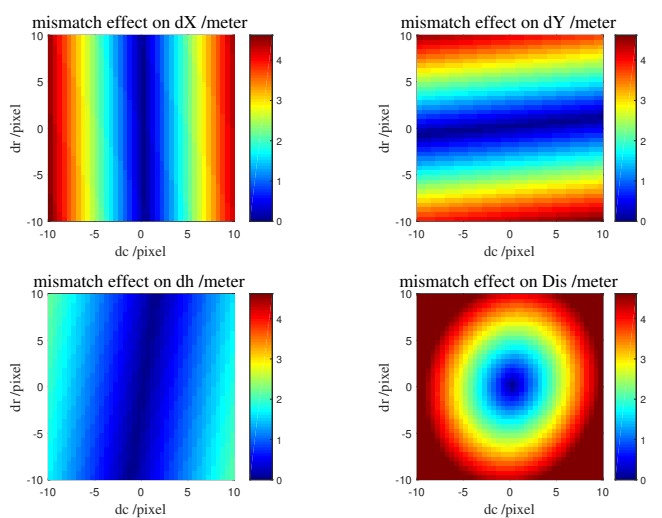

Figure 9. Illustration of the effect of mismatching a SAR keypoint in the optical image.

5 meters of absolute distance between the reconstructed object point and the correct object point. Comparing Fig. 9 and Tab. 2, the matching accuracy of the experiment conducted in this paper apparently can be up to \pm 5 pixels. However, it should be noted that the distances in Tab. 2 are prone to introduce a certain bias as well, as there may be situations where even the 3 or 10 nearest neighbors of the reconstructed point are a bad choice for evaluation.

Besides limiting the search space as suggested in this paper, another potential solution for enhancing the robustness of the matching procedure is to determine a higher threshold $\Delta S$, which only keeps the most reliable matches. How to determine $\Delta S$ in an unsupervised environment is part of ongoing research.

\subsection{Comment on Keypoint Selection}

Another possible shortage of the presented methodology is the utilization of the Harris corner detector, which certainly is not the perfect choice to detect significant keypoints in SAR imagery. This is illustrated in Fig. 10, which shows the Harris keypoints for the TUM main campus subset of the test area. A potential solution for this issue is to resort to SAR-specific interest point detection methods, e.g. based on point target analysis.

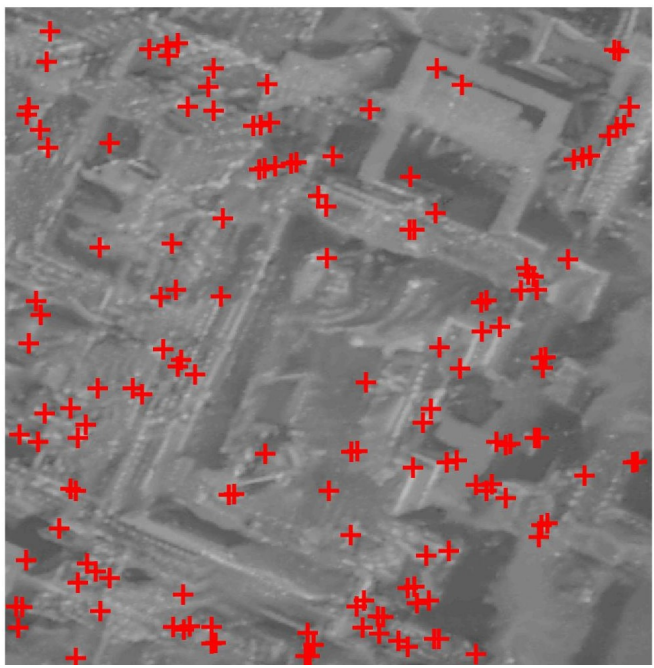

Figure 10. The feature points detected in the SAR image patch in the TUM main campus area.

\section{CONCLUSION AND OUTLOOK}

This paper presents a matching strategy for sparsely distributed tie points in the context of SAR-optical stereogrammetry. It shows that the major challenge for very-high-resolution imagery of densely built-up urban areas is a proper restriction of the search space, as mismatching errors directly propagate into erroneous $3 \mathrm{D}$ reconstructions. In order to enhance the robustness in tie point matching, a line-shaped search window was proposed, which enables a reconstruction accuracy in the meter-domain by simultaneously solving the matching and the $3 \mathrm{D}$ reconstruction problems, even though only simple normalized cross-correlation was used as a similarity measure. Future work will include investigations on more sophisticated similarity measures, as well as a keypoint detection procedure which is specifically tailored to SAR image characteristics. In addition, potential errors in the sensor imaging parameters will be considered in a multi-sensor bundle adjustment framework.

\section{ACKNOWLEDGEMENTS}

The authors want to thank European Space Imaging for providing the Worldview-2 image. Futhermore, they want to thank Gerald Baier of TUM-SiPEO for filtering the TerraSAR-X data.

This work is supported by the Helmholtz Association under the framework of the Young Investigators Group SiPEO (VH-NG1018, www.sipeo.bgu.tum.de) and the German Research Foundation (DFG), grant SCHM 3322/1-1.

\section{REFERENCES}

Bloom, A. L., Fielding, E. J. and Fu, X.-Y., 1988. A demonstration of stereophotogrammetry with combined SIR-B and landsat tm images. International Journal of Remote Sensing 9(5), pp. 1023-1038.

Capaldo, P., Crespi, M., Fratarcangeli, F., Nascetti, A. and Pieralice, F., 2012. DSM generation from high resolution imagery: applications with WorldView-1 and Geoeye-1. Italian Journal of Remote Sensing 44(1), pp. 41-53.

Deledalle, C.-A., Denis, L., Tupin, F., Reigber, A. and Jäger, M., 2015. Nl-sar: A unified nonlocal framework for resolutionpreserving (pol)(in) sar denoising. IEEE Transactions on Geoscience and Remote Sensing 53(4), pp. 2021-2038.

Gutjahr, K., Perko, R., Raggam, H. and Schardt, M., 2014. The epipolarity constraint in stereo-radargrammetric dem generation. IEEE transactions on geoscience and remote sensing 52(8), pp. 5014-5022.

Hebel, M. and Stilla, U., 2007. Automatic registration of laser point clouds of urban areas. International Archives of the Photogrammetry, Remote Sensing and Spatial Information Sciences 36(3/W49A), pp. 13-18.

Krieger, G., Moreira, A., Fiedler, H., Hajnsek, I., Werner, M., Younis, M. and Zink, M., 2007. TanDEM-X: A satellite formation for high-resolution SAR interferometry. IEEE Transactions on Geoscience and Remote Sensing 45(11), pp. 3317-3341.

Raggam, J., Almer, A. and Strobl, D., 1993. Multisensor mapping using SAR in conjunction with optical data. International Archives of the Photogrammetry, Remote Sensing and Spatial Information Sciences 29, pp. 556-556. 
Raggam, J., Almer, A. and Strobl, D., 1994. A combination of SAR and optical line scanner imagery for stereoscopic extraction of 3-d data. ISPRS Journal of Photogrammetry and Remote Sensing 49(4), pp. 11-21.

Raggam, J. and Almer, A., 1990. Mathematical aspects of multisensor stereo mapping. In: Proc. of IEEE International Geoscience and Remote Sensing Symposium, pp. 1963-1966.

Schmitt, M., 2014. Reconstruction of urban surface models from multi-aspect and multi-baseline interferometric SAR. PhD thesis, Universitätsbibliothek der TU München.

Schmitt, M. and Zhu, X., 2016a. On the challenges in stereogrammetric fusion of sar and optical imagery for urban areas. International Archives of the Photogrammetry, Remote Sensing and Spatial Information Sciences 41(B7), pp. 719-722.

Schmitt, M. and Zhu, X. X., 2016b. Data fusion and remote sensing: An ever-growing relationship. IEEE Geoscience and Remote Sensing Magazine 4(4), pp. 6-23.

Tao, C. V. and Hu, Y., 2001. A comprehensive study of the rational function model for photogrammetric processing. Photogrammetric engineering and remote sensing 67(12), pp. 1347-1358.

Xing, S., Xu, Q., Zhang, Y., He, Y. and Jin, G., 2008. Opti$\mathrm{cal} / \mathrm{SAR}$ sensors stereo positioning. International Archives of the Photogrammetry, Remote Sensing and Spatial Information Sciences 37(B1), pp. 993-996.

Ye, Y., Shan, J., Bruzzone, L. and Shen, L., 2017. Robust registration of multimodal remote sensing images based on structural similarity. IEEE Transactions on Geoscience and Remote Sensing $\mathrm{PP}(99)$, pp. 1-18.

Zhang, H., Xu, H. and Wu, Z., 2015. A novel fusion method of SAR and optical sensors to reconstruct 3-D buildings. In: Proc. of IEEE International Geoscience and Remote Sensing Symposium, pp. 609-612.

Zhang, Z., Deriche, R., Faugeras, O. and Luong, Q.-T., 1995. A robust technique for matching two uncalibrated images through the recovery of the unknown epipolar geometry. Artificial intelligence 78(1-2), pp. 87-119. 